\title{
Review of: "The impact of free vaccination policies under the Korean Influenza National Immunization Program: Trends in influenza vaccination rates in South Korea from 2010 to 2019"
}

\author{
Akihiko Saitoh ${ }^{1}$ \\ 1 Niigata University
}

Potential competing interests: The author(s) declared that no potential competing interests exist.

Seo and Lim et al . conducted a cross-sectional study with nationwide survey data in Korea for influenza vaccination rates of the following four target groups: children $\leq 12$ years, adults $\geq 65$ years, pregnant women, and people with chronic diseases between 2010 and 2019. In total, 80,861 individuals were analyzed. From 2017 to 2019, the vaccination coverage of children $\leq 12$ years, pregnant women improved, and the elderly $\geq 65$ years showed the highest rates, while people with chronic diseases marked the lowest. They concluded that the recent expansion of financial aids to children $\leq 12$ years and pregnant women was followed by significant increases in vaccination rates in both groups. They suggest that free vaccination policy is one of the most effective strategies to enhance vaccination coverage, and they call for its expansion to other under-vaccinated target groups, especially people with chronic diseases. Overall, the manuscript summarizes available data well, and emphasize the importance of free cost to improve vaccination rates.

Major

$1 \square$ Among the children $\leq 12$ years, how were the vaccination rates in different ages, e.g. infants, 1-2 years, 3-6 years, 7-12 years, etc. This reviewer would like to know how the vaccination rates differ in different age groups in children.

$2 \square$ What was the overall const-effectiveness of this influenza vaccine intervention in Korea?3ฤHow do pregnant women accept influenza vaccination in Korea? Do they understand the risks and benefits well about the vaccine? 